\title{
Treatment outcome of clonal cytopenias of undetermined significance: a single-institution retrospective study
}

\author{
Zhuoer Xie $\mathbb{1}^{1}$, Ahmad Nanaa', Antoine N. Saliba', Rong He², David Viswanatha ${ }^{3}$, Phuong Nguyen², \\ Dragan Jevremovic (103), Patricia Greipp², Mohamad E. Salama², Naseema Gangat [1', Hassan B. Alkhateeb (1)', \\ Ayalew Tefferi ${ }^{1}$, Mark Litzow ${ }^{1}$, Mrinal Patnaik (10)', Mithun Shah (1) ${ }^{1}$ and Aref Al-Kali (1) ${ }^{1}$
}

Dear Editor,

Clonal cytopenia(s) of undetermined significance (CCUS) is defined by the presence of the somatic mutation(s) of genes associated with myeloid neoplasms, with unexplained cytopenia(s) but without definitive morphologic evidence of myeloid neoplasms ${ }^{1,2}$. CCUS is a newly described entity with a high probability of progression to myeloid disorders upon follow-up ${ }^{3,4}$. However, there is a paucity of data on the efficacy of treatment ${ }^{5}$. We hereby describe a single-institution's experience in managing CCUS patients who required treatment beyond blood transfusions.

This retrospective study was approved by scientific and ethical review boards at our institution. Patients who had CCUS and had received treatment beyond blood transfusions were identified from medical records. CCUS diagnosis was confirmed based on the absence of definitive morphologic evidence of myeloid neoplasms from bone marrow biopsy evaluation combined with evidence of pathogenic myeloid somatic mutation with a variant allele frequency (VAF) of at least $2 \%$ using our institution's next-generation sequencing (NGS) panel (OncoHeme, Mayo Clinic) ${ }^{6}$. Treatment type and the indication were based on the treating physician's choice. Clinical and laboratory results were collected. Hematologic improvement (HI) was graded based on the MDS International Working Group (IWG) 2006 criteria $^{7}$. Symptomatic improvement (SI) was defined by the subjective

\footnotetext{
Correspondence: Aref Al-Kali (alkali.aref@mayo.edu)

'Division of Hematology, Mayo Clinic, Rochester, MN, USA

2Department of Laboratory Medicine and Pathology, Mayo Clinic, Rochester, MN, USA
}

Full list of author information is available at the end of the article improvement of CCUS-related inflammatory symptoms based on the patients' report. The treatment response rate (RR) was calculated based on HI and SI. Progression was defined by either the occurrence of myeloid neoplasm or worsening cytopenia(s). Dependence on blood transfusion was defined as requiring an average of $\geq 2$ units of packed red blood cells (RBCs) or platelets over 4 weeks or $\geq 4$ units over 8 weeks. The date of treatment initiation was used for calculating overall survival (OS) and progressionfree survival (PFS). Stata 14.1 (StataCorp) was used for data analysis.

Between July 2015 and July 2020, 24 patients met the inclusion criteria with a median age of 72 years (range: 24-87). The majority of patients were males $(N=20$, $83 \%)$. Three $(13 \%)$ patients had a history of the hematological condition (one monoclonal B cell lymphocytosis [MBL], one congenital neutropenia, and one acute myeloid leukemia [AML]) in remission). Three (13\%) had a history of solid organ malignancy (one bladder cancer, one bronchogenic carcinoma, and one lung cancer). Two (8\%) patients had prior radiation therapy, two (8\%) had prior chemotherapy, and one (4\%) had prior allogeneic stem cell transplantation. Six (25\%) patients had inflammatory conditions (one Sweet's syndrome, one periodic fever syndrome, one systemic inflammatory response syndrome, one poly-inflammatory syndrome with vasculitis, one myopericarditis, and one inflammatory purpura) (Table 1 and Supplemental Table 1). At the time of treatment, median hemoglobin was $8.9 \mathrm{~g} / \mathrm{dl}$ (range 6.8-13.7), white blood cell $2.9 \times 10^{9} / 1$ (range 1.4-12.3), and platelet $76 \times 10^{9} / 1$ (range $8-407$ ). RBC- and platelet transfusion-dependent rates were $54 \%$ and $29 \%$, respectively. We identified 21 different mutations. Most 


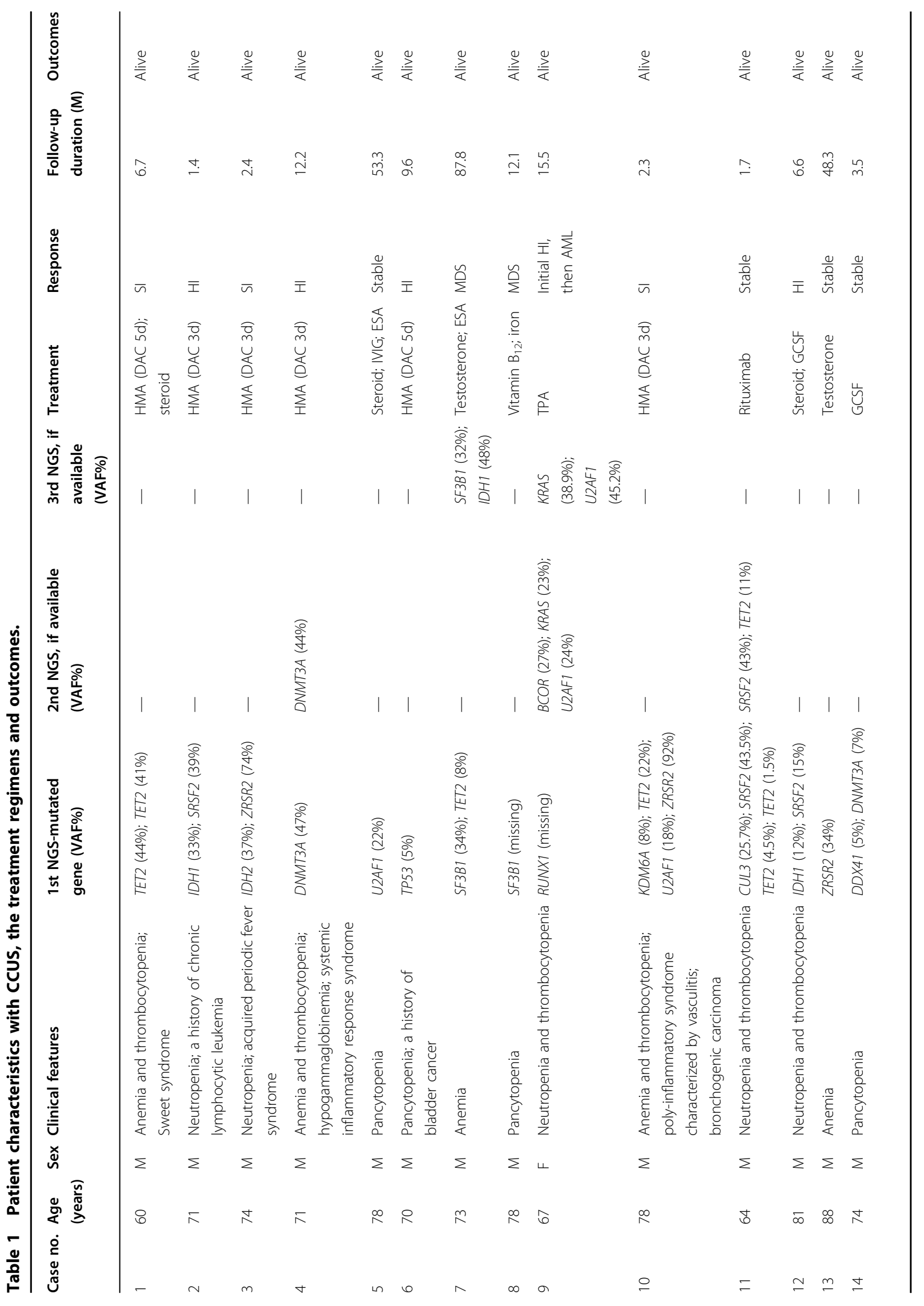




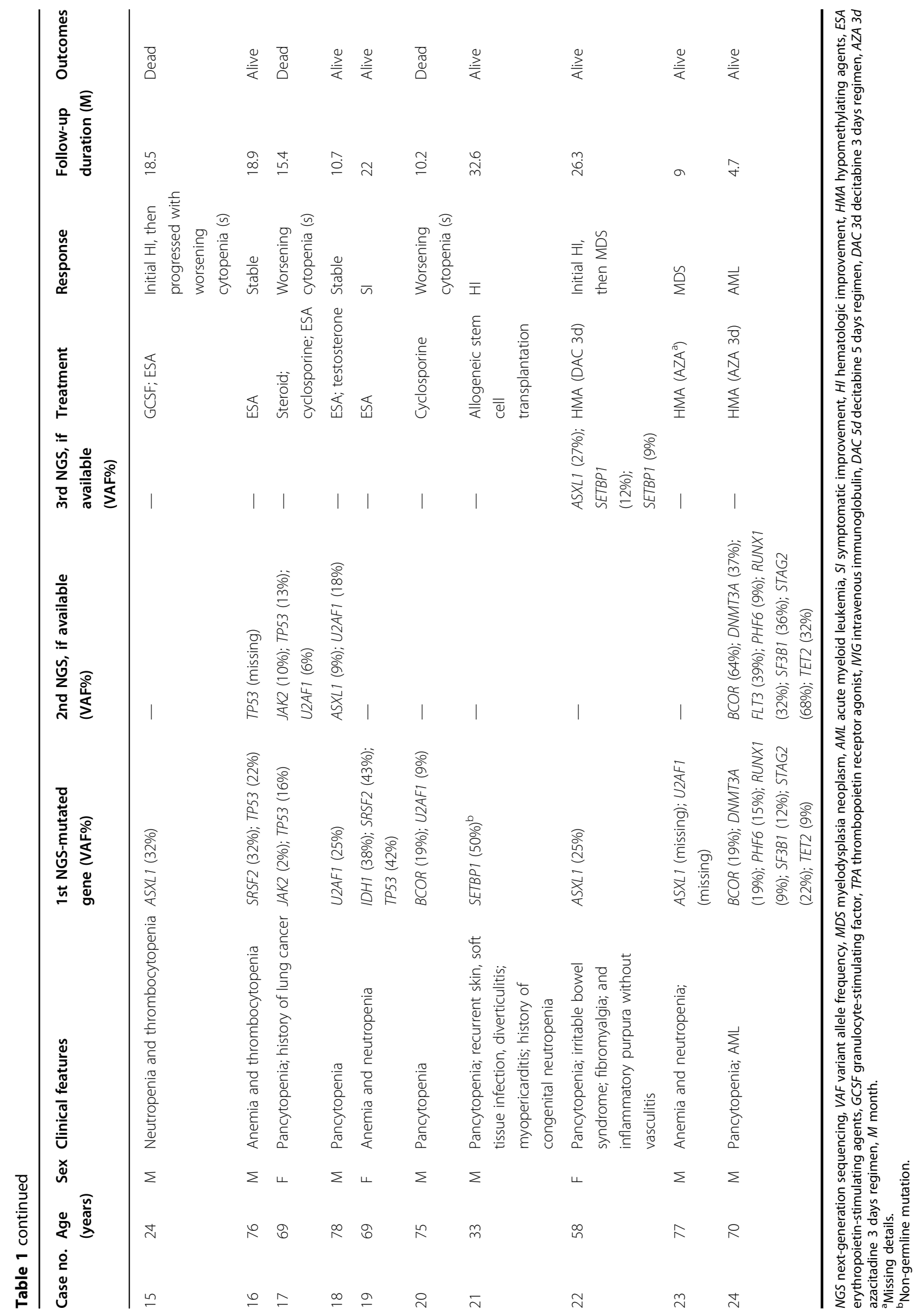




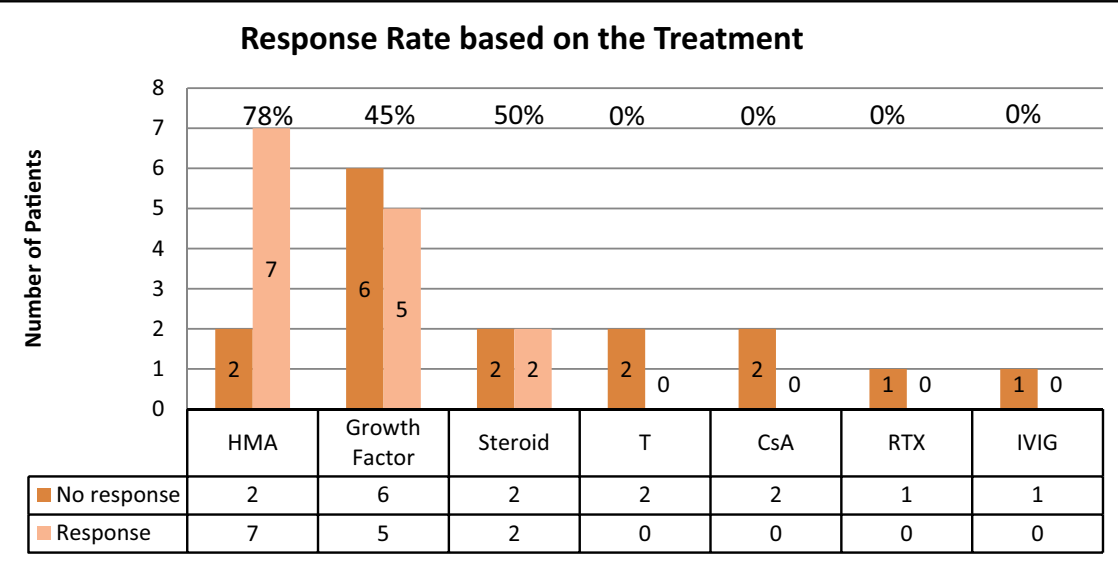

Fig. 1 Disease response rate (RR). Number and percentage of responders based on the treatment types. HMA hypomethylating agents, T testosterone, CsA cyclosporin, IVIG intravenous immunoglobulin, RTX rituximab.

common mutations occurred in SRSF2 $(N=5,21 \%)$, TET2 $(N=5,21 \%)$, U2AF1 $(N=5,21 \%)$, and TP53 $(N=$ 4, 17\%) (Supplemental Fig. 1). This is consistent with previous reports that these genes are commonly seen in clonal hematopoiesis of indeterminate potential or CCUS $^{8,9}$. Ten (42\%) patients had 1 mutation, and 14 (58\%) had $\geq 2$ mutations. The median VAF was $25.7 \%$ (range 1.5-92\%) (Supplemental Table 2).

Treatment included hypomethylating agents (HMAs) $(N=9,38 \%$ [six treated with 3 days regimen], growth factors $(N=11,46 \%)$ [erythropoietin-stimulating agents (ESA) $(N=7,29 \%)$, granulocyte-stimulating factor) $(N$ $=3,13 \%)$ and thrombopoietin receptor agonist $(N=1$, $4 \%)$, steroids $(N=4,17 \%)$, testosterone $(N=2,8 \%)$, cyclosporine $(N=2,8 \%)$, rituximab $(N=1,4 \%)$, intravenous immunoglobulin $(N=1,4 \%)$, and vitamin $\mathrm{B}_{12}$ and iron $(N=1,4 \%)$. Seven (29\%) patients received $\geq 2$ different agents. The median time from diagnosis to treatment was 2.1 months (range $0-26.8$ ) with a median follow-up duration of 14.3 months (range 2.3-59.9). The overall best RR was 50\% (HI: $N=8(33 \%)$ and SI: $N=4$ $(17 \%))$. Three (13\%) patients subsequently progressed after the initial response. Most responders (58\%) were treated with HMA ( $\mathrm{RR}=78 \%, p=0.04$ [HI: $N=3$, RR $=57 \%$ and SI: $N=3, \mathrm{RR}=43 \%]$ ) with a median response duration of 6.8 months (range: 1.4-12.4) (Supplemental Figure 2). HMA RR was not associated with mutations in DNA methylation genes $(p>0.05)$. Among the patients with inflammatory conditions, five were treated with HMA, and the SI-RR was $60 \%$. The RR was $100 \%$ for the three patients with $I D H 1$ mutation (treated with HMA, steroids, and ESA, respectively). The RR was $67 \%$ for patients with ZRSR2 mutation (two HMA, one testosterone). The RR was $0 \%$ for three patients with $S F 3 B 1$ mutation (one testosterone and ESA, one vitamin $B_{12}$ and iron, and one HMA) (Table 1).
None of the responders had $A S X L 1$ mutation. Figure 1 demonstrates the RR for each treatment. Patients with a single mutation (compared to $\geq 2$ mutations) were significantly more likely to achieve HI (RR 60 vs. $14 \%, p=$ $0.03)$. At the data cutoff, RBC and platelet transfusiondependent rate were $38 \%$ (30\% reduction) and 25\% (14\% reduction), respectively.

Six patients (25\%) progressed to myeloid malignancy (four MDS and two AML). Five (21\%) had worsening cytopenias. Among the four MDS patients, they had SF 3B1, TET2, ASXL1, and U2AF1 mutations at the diagnosis of CCUS. Within the two AML patients, both had RUNX1 mutation (Table 1 and Supplemental Table 3). A recent report from Malcovati et al. ${ }^{10}$ suggests that SF3B1 mutation in CCUS represents a distinct MDS entity. All three SF3B1 mutant CCUS patients progressed to myeloid neoplasms in this cohort. Two patients with a prior diagnosis of solid organ malignancies (treated with cytotoxic chemotherapy) had CCUS with TP53 mutation. One patient treated with HMA achieved HI, while the other, treated with steroids, cyclosporine, and ESA, did not respond. The median PFS was 17.1 months (95\% CI: 7.1-not reached). The median OS was not reached with an estimated 2-year OS of 73\% (Supplemental Figure 3A, B). Three (13\%) patients had worsening cytopenias leading to death (one septic shock, two transitioned to hospice care). These three patients' molecular signature at CCUS diagnosis showed ASXL1, TP53, and U2AF1 mutations, respectively (Table 1 and Supplemental Table 4). The 30and 60 -day mortality was $0 \%$.

Subsequent NGS was available for nine (38\%) patients. Six $(66.7 \%)$ had a clonal evolution (acquiring new mutations). Among these, two progressed to AML (one had new $B C O R, K R A S$, and $U 2 A F 1$ mutations, one had new FLT3 mutation); two progressed to MDS (one had new IDH1 mutation, one had new SETBP1 mutation); one had 
worsening cytopenia (new U2AF1 mutation); one had stable disease (new ASXL1 mutation); two had stable disease with no clonal evolution; one responded to HMA treatment with the DNMT3A VAF decreasing from 47 to 44\% (Table 1 and Supplemental Table 3). In our cohort, five $(83 \%)$ clonal evolution co-occurred with the progression of myeloid neoplasm.

In summary, this is the first report of the outcome of treatment in CCUS patients requiring therapies beyond blood transfusion. Our results suggest that patients with CCUS responded to available treatments for myeloid disorders and immune cytopenias. HMA was safe and associated with the highest RR and CCUS-associated inflammatory symptoms in this cohort. Our data also suggest that the mutation profile may impact treatment response. Several limitations exist in our study. First, the retrospective nature of our cohort and the relatively small sample size limit the generalizability of our observations. Second, there were heterogeneities in both patients and therapies. Three patients with pre/coexisting hematological conditions (one MBL, one congenital neutropenia who received stem cell transplantation for pancytopenia, and one AML in remission) were included as they did not fulfill myeloid malignancy criteria. Several patients were diagnosed with inflammatory disorders or malignancies and received prior cytotoxic chemotherapy making them a higher risk CCUS. Although CCUS is associated with high inflammatory status ${ }^{11}$, it is unclear if the immune-mediated marrow process is the driver of cytopenia or the clonal process itself. The therapies also varied based on the treating physician's decision and were adopted from myeloid disorder or immune-related cytopenia as no standard of care exists to date. Third, the best treatment response was defined as both hematological and symptomatic responses due to the unique features of this cohort, with inflammatory status present in a quarter of our patients. Last, the MDS IWG criteria were used to assess hematological response for this cohort due to a lack of other criteria. An international multicenter study is ongoing to validate our current findings and determine the clinical outcome for treatment in CCUS. Future studies are warranted to develop response and progression criteria to facilitate and standardize reporting treatment outcomes.

\section{Author details}

${ }^{1}$ Division of Hematology, Mayo Clinic, Rochester, MN, USA. ${ }^{2}$ Department of Laboratory Medicine and Pathology, Mayo Clinic, Rochester, MN, USA. ${ }^{3}$ Department of Hematopathology, Mayo Clinic, Rochester, MN, USA

\section{Author contributions}

Z.X. and A.A. designed the study, interpreted the data, and wrote the manuscript; Z.X. collected the data and conducted the statistical analysis; A.N.S., N.G., H.B.A., A.T., M.L., M.P., M.S., and A.A. cared for the patients and provided patients' information; A.N., R.H., D.V., P.N., D.J., P.G., and M.E.S. performed the next-generation sequencing; and all authors critically reviewed and approved the manuscript.

\section{Conflict of interest}

The authors declare no competing interests.

\section{Publisher's note}

Springer Nature remains neutral with regard to jurisdictional claims in published maps and institutional affiliations.

Supplementary information The online version contains supplementary material available at https://doi.org/10.1038/s41408-021-00439-x.

Received: 28 November 2020 Revised: 2 February 2021 Accepted: 5 February 2021

Published online: 01 March 2021

\section{References}

1. Bejar, R. CHIP, ICUS, CCUS and other four-letter words. Leukemia 31, 1869-1871 (2017).

2. Steensma, D. P. et al. Clonal hematopoiesis of indeterminate potential and its distinction from myelodysplastic syndromes. Blood 126, 9-16 (2015).

3. Malcovati, L. et al. Clinical significance of somatic mutation in unexplained blood cytopenia. Blood 129, 3371-3378 (2017).

4. Cargo, C. A. et al. Targeted sequencing identifies patients with preclinical MDS at high risk of disease progression. Blood 126, 2362-2365 (2015).

5. Steensma, D. P. Clinical implications of clonal hematopoiesis. Mayo Clin. Proc. 93, 1122-1130 (2018).

6. He, R. et al. Hybridization capture-based next generation sequencing reliably detects FLT3 mutations and classifies FLT3-internal tandem duplication allelic ratio in acute myeloid leukemia: a comparative study to standard fragment analysis. Mod. Pathol. 33, 334-343 (2020).

7. Cheson, B. D. et al. Clinical application and proposal for modification of the International Working Group (IWG) response criteria in myelodysplasia. Blood 108, 419-425 (2006).

8. Kwok, B. et al. MDS-associated somatic mutations and clonal hematopoiesis are common in idiopathic cytopenias of undetermined significance. Blood 126, 2355-2361 (2015).

9. Xie, M. et al. Age-related mutations associated with clonal hematopoietic expansion and malignancies. Nat. Med. 20, 1472-1478 (2014).

10. Malcovati, L. et al. SF3B1-mutant MDS as a distinct disease subtype: a proposal from the International Working Group for the Prognosis of MDS. Blood 136, 157-170 (2020)

11. Franceschi, C. \& Campisi, J. Chronic inflammation (Inflammaging) and its potential contribution to age-associated diseases. J. Gerontol. A 69, S4-\$9 (2014). 